\title{
Future roles for FoxM1 inhibitors in cancer treatments
}
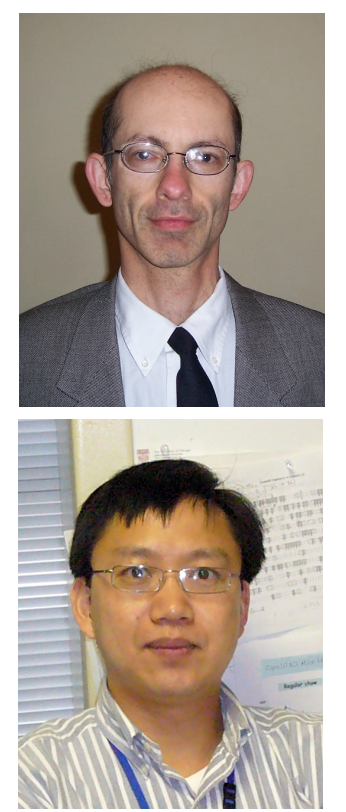

Guy R Adami \& Honggang Ye

${ }^{\dagger}$ Author for correspondence University of Illinois at Chicago, Department of Oral Medicine \& Diagnostic Sciences, 801 S. Paulina Street, M/C 838, Chicago IL 60612, USA

Tel.: +1 3129966251 ; Fax: +1312355 2688; gadami@uic.edu



'A prototype high-throughput screen for FoxM1 inhibitors has been useful and has produced one potential compound - Siomycin A.'

Targeting tumor cells based on their high levels of proliferation has long been an important component of cancer treatment. Historically, oncologists have capitalized on the difference in proliferation rates of normal and tumor cells by treating patients with chemicals that damage replicating DNA and or the proteins that segregate the chromosomes of proliferating cells, thus preferentially killing tumor cells. Knowledge regarding the proteins in cells that regulate the steps of the cell cycle and the proteins that regulate these regulators has now increased. Forkhead box (Fox)M1 is a nuclear protein that turns on the expression of a long list of proteins and enzymes required for mitosis and cytokinesis, and is an example of a master cell-cycle regulator [1]. Since rapid traverse of the cell cycle is an important characteristic of at least some cells of almost all malignant tumors, it would suggest that tumor cells must produce large amounts of FoxM1 or find a way to proliferate without it. Apparently the former mode is favored as FoxM1 enrichment is one of the most consistent tumor markers among solid tumors derived from prostate, breast, lung, ovary, colon, pancreas, stomach, bladder, liver and kidney [2]. This would suggest that an alternative approach to cancer chemotherapy would be to inhibit FoxM1, a master regulator of the cell cycle.

\section{FoxM1 inhibition kills or blocks tumor cells from proliferating}

Inhibition of FoxM1 in tumor cell lines grown in vitro results in rapid apoptotic cell death or permanent cell cycle arrest [3-7]. Even more remarkably, genetic removal of FoxM1 is a tumor blocker in rodent cancer models. Transgenic mice, which were constructed to ablate the FoxM1 gene in hepatocytes by 6 weeks after birth, demonstrated resistance to the induction of hepatocellular carcinoma after carcinogen exposure [8]. Similarly, other transgenic adult mice, with only $25 \%$ of pulmonary cells retaining the FoxM1 gene, demonstrated substantial inhibition of squamous cell carcinoma and adenocarcinoma induced by long-term exposure to the environmental carcinogen, urethane [7]; the inhibition occurred both on the level of tumor formation and progression. This is in contrast to the deletion of another oncogene - jun - which, when deleted after tumor growth, is no longer anticarcinogenic [9]. One caveat is that FoxM1 has long been thought to be crucial in allowing healing of normal injured tissue [1]. It is important to examine whether FoxM1 inhibition will block tumors without harming normal tissue. To date, FoxM1 has been tested in two organs and has passed with flying colors. Genetic deletion of the gene in the models above does not result in morbidity over many months $[7,8]$. Additionally, specific tests in response to liver surgery show that, while some steps of the healing process take longer in old mice where FoxM1 expression is normally very low, the overall process still occurs [10]. It will be important to know more about the specificity of the effects of FoxM1 deprivation on normal tissue in the adult; however, based on what is currently known, even long-term inhibition of FoxM1 will have minimal toxicity in many organs.

\section{Future application of FoxM 1 inhibition to} the clinic

Methods used in vitro to temporarily shut off the FoxM1 gene or to block the protein function are varied and, not surprisingly, somewhat limited in their applicability to inhibiting FoxM1 in vivo. First, activity can be efficiently decreased by a peptide inhibitor [8]. This peptide is a miniature version of the alternative reading frame (ARF) tumor-suppressor protein, which appears to be a natural inhibitor of the FoxM1 protein, binding to FoxM1 in the nucleus and inactivating it. The miniature version of ARF given therapeutically would take over in cancer cells when the endogenous ARF protein is mutated or 'switched' off. As with most peptide drugs that need to move inside cells to find their targets, the mini-ARF protein has to be modified through the addition of extra peptides in order to allow it to cross cell membranes. While the modification of polypeptides to allow cell uptake has been successful in vitro, and despite the fact that there have been 
a few positive results with the liver as a target in vivo, many problems, including organ- and cell-targeting of polypeptides, severely limit the clinical potential of this approach in the near future [11,12]. This is in contrast to peptide inhibitors of extracellular proteins, such as monoclonal antibodies to cell-receptor proteins, which are currently being used in tumor therapy.

'The most promising method of FoxM1 inhibition is small molecule inhibition.'

The second standard cell-culture methodology applied to the blocking of FoxM1 production is the gene therapy approach of using small interfering (si)RNA oligonucleotides to FoxM1. In culture, the use of lipid vesicles to increase siRNA uptake into cells resulted in decreased FoxM1 protein synthesis and cell death, or irreversible cell cycle arrest for a number of cancer cell lines [3-5]. In recent years, there has been significant progress made in improving methods of efficient uptake of such oligonucleotides into the cells of intact tissue in vivo, and many examples exist for the successful introduction of siRNA into cells in the body. Those who are skeptical owing to similar optimism about antisense approaches to inhibition 15 years ago should understand that in the laboratory siRNA works much more consistently than antisense ever did. We therefore have every reason to think it will work better in the clinic. Whether or not it can be applied to FoxM1 as a tumor therapy will depend on two things. First, as in most successful tumor therapies that do not hit all cells, there will be a requirement for a bystander effect - even cells that do not take up the siRNA will have to die. Alternatively, it will have to be used alongside a complementary therapy, such as surgery, which removes many of the cells that do not take up the siRNA.

The most promising method of FoxM1 inhibition is small molecule inhibition. Highthroughput analysis allows the rapid screening for compounds that block FoxM1 transcriptional activation in a relatively specific manner. This approach generates fewer false positives compared with screening methods that are based on tumorcell killing, and is an efficient way to generate potential drugs that kill or block the proliferation of tumor cells without killing normal cells. This approach has already started to show promising results. Gartel, Costa and coworkers have designed a cell line that expresses high levels of tagged FoxM1, which, in turn, regulates the expression of an encoded reporter protein [13].
Both proteins are easily quantitated. A screen of the National Cancer Institute challenge and diversity set of approximately 2000 compounds revealed one hit - Siomycin A - an antibiotic. They demonstrated that this antibiotic inhibits cyclin-dependent kinase (CDK) phosphorylation required for activation of FoxM1 in each cell cycle, and it causes tumor cell death in vitro. This screen appears to answer the question of whether examining small molecules that target FoxM1 is a viable option. In the near future, it should be producing compounds for clinical trials. Indeed, drugs currently in the clinic or clinical trials, such as the CDK inhibitor roscovitine, may be shown to kill tumor cells via FoxM1 inhibition [14].

So where will we be in 10 years with regard to FoxM1 and cancer drug therapy? It is important to repeat that FoxM1 was originally heralded as a protecting protein that enhanced liver regeneration/lung regeneration after tissue and cell damage $[1,15]$. The presence of FoxM1 inhibitors as part of a cancer treatment cocktail will almost certainly increase the killing of tumor cells by most standard genotoxic compounds. One obvious question that researchers appear afraid to examine is whether short-term FoxM1 inhibition will be toxic to normal, high-proliferating cell compartments of the adult. We know that FoxM1 inhibition or gene deletion in normal mouse embryo fibroblasts in vitro is toxic and mutagenic [4]. But in vitro these cells are not genetically stable to begin with. An experiment to test the effect of FoxM1 inhibition on the gastrointestinal tract and the hematopoietic system in the adult mouse is required. Will transient loss of FoxM1 cause rapid runaway cell death or irreversible cell cycle arrest? And how mutagenic will it be? We would suggest that some cell death will occur but long-term mutagenesis will be minimal. If so, inhibition of FoxM1 will be a useful strategy for cancer therapy. If not, undoubtedly other members of the cell-proliferation pathway that FoxM1 regulates, such as Polo-like kinase 1, will be prime potential cancer therapy targets, and it will be necessary to learn more about the mechanisms through which FoxM1 acts [16].

\section{Future perspective}

A prototype high-throughput screen for FoxM1 inhibitors has been useful and has produced one potential compound - Siomycin A. However, we need to know more about the effects of FoxM1 inhibition on the rapidly proliferating normal tissues of the adult patient. 


\section{Executive summary}

- A master regulator of the cell cycle is required in tumor cells.

- FoxM1 enrichment in tumor cells promotes the transcription of cell cycle promoting enzymes, and is required for tumor cell proliferation in vivo.

- FoxM1 inhibition either kills or irreversibly blocks tumor cells from proliferating.

- An alternative reading frame miniprotein that binds FoxM1 in cells blocked liver tumor formation and growth.

- Short interfering RNA to FoxM1 causes tumor cell death or irreversible cell cycle arrest in vitro.

- An antibiotic that blocks FoxM1 phosphorylation blocks FoxM1 activity and kills tumor cells in vitro.

\section{Bibliography}

Papers of special note have been highlighted as either of interest $(\bullet)$ or of considerable interest $(\bullet \bullet)$ to readers.

1. Laoukili J, Stahl M, Medema RH: FoxM1: at the crossroads of ageing and cancer. Biochim. Biophys. Acta 1775 (1), 92-102 (2006).

- Recent review that outlines the genes and pathways regulated by Forkhead box (Fox)M1.

2. Pilarsky C, Wenzig M, Specht T, Saeger HD, Grutzmann R: Identification and validation of commonly overexpressed genes in solid tumors by comparison of microarray data. Neoplasia 6 , 744-750 (2004).

3. Wonsey DR, Follettie MT: Loss of the forkhead transcription factor FoxM1 causes centrosome amplification and mitotic catastrophe. Cancer Res. 65, 5181-5189 (2005).

4. Laoukili J, Kooistra MR, Bras A et al:: FoxM1 is required for execution of the mitotic programme and chromosome stability. Nat. Cell Biol. 7, 126-136 (2005).

5. Wang IC, Chen YJ, Hughes D et al:: Forkhead box M1 regulates the transcriptional network of genes essential for mitotic progression and genes encoding the SCF (Skp2-Cks1) ubiquitin ligase. Mol. Cell. Biol. 25, 10875-10894 (2005).

6. Kalin TV, Wang IC, Ackerson TJ et al: Increased levels of the FoxM1 transcription factor accelerate development and progression of prostate carcinomas in both TRAMP and LADY transgenic mice. Cancer Res. 66, 1712-1720 (2006).
7. Kim IM, Ackerson T, Ramakrishna $S$ et al:: The Forkhead Box M1 transcription factor stimulates the proliferation of tumor cells during development of lung cancer. Cancer Res. 66, 2153-2161 (2006).

8. Kalinichenko VV, Major ML, Wang X et al:: FoxM1b transcription factor is essential for development of hepatocellular carcinomas and is negatively regulated by the p19ARF tumor suppressor. Genes Dev. 18, 830-850 (2004).

- First indication that FoxM1 inhibition blocks tumor formation (hepatocellular carcinoma) in vivo using an animal model. There was a lack of normal liver toxicity over several months.

9. Eferl R, Ricci R, Kenner L et al.: Liver tumor development. c-Jun antagonizes the proapoptotic activity of p53. Cell 112 , 181-192 (2003).

10. Wang X, Krupczak-Hollis K, Tan Y, Dennewitz MB, Adami GR, Costa RH: Increased hepatic Forkhead Box M1B (FoxM1B) levels in old-aged mice stimulated liver regeneration through diminished p27Kip1 protein levels and increased Cdc25B expression. J. Biol. Chem. 277, 44310-44316 (2002).

11. Walensky LD, Kung AL, Escher I et al: Activation of apoptosis in vivo by a hydrocarbon-stapled BH3 helix. Science 305, 1466-1470 (2004).

12. Gusarova GA, Wang IC, Major ML et al:: A cell-penetrating ARF peptide inhibitor of FoxM1 in mouse hepatocellular carcinoma treatment. J. Clin. Invest.117 (1), 99-111 (2006).
13. Radhakrishnan SK, Bhat UG, Hughes DE, Wang IC, Costa RH, Gartel AL:

Identification of a chemical inhibitor of the oncogenic transcription factor forkhead box M1. Cancer Res. 66, 9731-9735 (2006).

-. Prototype high-throughput screen to identify compounds that inhibit FoxM1 activity and kill tumor cells.

14. Blagden S, de Bono J: Drugging cell cycle kinases in cancer therapy. Curr. Drug Targets 6, 325-335 (2005).

15. Tan Y, Raychaudhuri P, Costa RH: Chk2 mediates stabilization of the FoxM1 transcription factor to stimulate expression of DNA repair genes. Mol. Cell. Biol. (2006).

16. Strebhardt K, Ullrich A: Targeting polo-like kinase 1 for cancer therapy. Nat. Rev. Cancer 6, 321-330 (2006).

\section{Affiliations}

\section{- Guy R Adami}

University of Illinois at Chicago,

Department of Oral Medicine \& Diagnostic

Sciences, 801 S. Paulina Street, M/C 838,

Chicago IL 60612, USA

Tel.: +1312996 6251;

Fax: +13123552688 ;

gadami@uic.edu

- Honggang Ye

University of Chicago, Department of Medicine,

Chicago, IL 60637, USA

Tel.: +1 7737029117 ;

Fax: +1773702 9237;

hye@uchicago.edu 